\title{
1. Meta-analysis and value transfer: comparative assessment of scientific knowledge
}

\author{
Raymond J.G.M. Florax, Peter Nijkamp, \\ Kenneth G. Willis
}

\section{THE KNOWLEDGE PARADOX IN SCIENTIFIC ANALYSIS}

The level of scientific knowledge has risen to an unprecedented degree in the postwar period and the cumulative stock of new knowledge has in the past fifty years far exceeded the total knowledge stock gathered by mankind during the previous centuries. Not surprisingly, our modern society is often referred to as a knowledge society. Notwithstanding this favourable picture, it is also increasingly recognized that the need for more information has risen almost limitlessly. From daily operational choices to long-term strategic decisions, the amount of information needed by decision makers can hardly be satisfied or covered anymore. It thus seems that in a knowledge society the demand for scientific knowledge and information is growing even faster than the supply. This mismatch, called the 'knowledge paradox', prompts the question whether the balance between supply and demand can be restored.

A surprising feature of the abundance of scientific insights in our current knowledge society is the lack of scientific synthesis. The process of scientific knowledge gathering proceeds usually in a fragmented and individualized way, without due attention to research efforts made previously or elsewhere. This is clearly witnessed by the present popularity of, and need for, survey articles that aim to review concisely the state of affairs in a relevant area of scientific research. Most of these contributions, useful as they are, are however based on literary approaches, and certainly not on more rigorous approaches for comparative or synthesizing analyses.

The fragmentation in the scientific knowledge acquisition process has led to the emergence of 'niches' or 'islands' of scientific knowledge, without a 
satisfactory - and fruitful - connection with scientifically related areas. Despite advances in artificial intelligence methods, cognitive evolutionary approaches and quantum computing, comprehensiveness has become an illusion in many disciplines and even the integration of knowledge is scarcely feasible. This observation certainly applies to interdisciplinary research, but even in intradisciplinary research the level of free communication and cooperation, let alone integration, is feeble and problematic. This situation is not a coincidental development in the knowledge collection process, but is closely linked to the history of science philosophy in the Western world.

In the past few centuries the major impact on the scientific research methodology has been exerted by Cartesian philosophy, where analysis has become the central focus. Analysis means a breakdown of a complex reality into constituents that may be subjected to substantive and causal scientific investigation. The concentration on subsets of a complex constellation (made up of elements and relationships that exist in the real world) has certainly generated many important insights into separate phenomena (see Bal and Nijkamp, Chapter 3 of the present volume; Button et al., 1999). Remaining uncertainty was next tackled by a further fragmentation and analysis of relevant components of a phenomenon, without due attention to contextual factors which might have been identified through a consideration of related research efforts elsewhere. This constantly repetitive, deductive research methodology has led to a wealth of insights into details, but less so to major links within and between similar phenomena. As a consequence, a need has arisen to investigate the behaviour of complex systems by using principles from synergetics. This situation may also explain the current popularity of chaos theory, which attempts to study turbulence conditions in deterministic non-linear dynamic systems by infinitesimal changes in initial conditions (Nijkamp and Reggiani, 1998). In any case, conclusive answers are usually difficult to find, and beyond the horizon of a tentative finding on a fragment of reality, a new - usually even more detailed - research question appears to emerge.

In recent years, owing to improved communication among scientists in a certain discipline (through professional networks, the Internet, exchange of databases), the climate for a better exchange of knowledge has become more favourable as the documentation, collection and retrieval of study results have become easier. Nevertheless, the present situation still leaves much to be desired and inconsistencies (or large variations) between study findings are still more a rule than an exception. Hence there is certainly a need for more context-specific research in a comparative sense, but this requires a serious effort and more research synthesis based on (quasi-) experimentation. 
In empirical research, we also may often observe statistical biases emerging from small samples, measurement errors and biased data, so that, indeed, empirical comparative analyses on distinct case studies may suffer from biases too. The only meaningful way to overcome this cumbersome situation is to develop a proper - preferably quantitatively oriented - methodology for a systematic comparison and synthesis of previously obtained analytical knowledge from in-depth (case) studies. Against this background, meta-analytical methods may offer a promising new departure (see van den Bergh et al., 1997). Such an effort may lead to a more satisfactory performance of research approaches, as it may be helpful to distinguish between phenomenon-intrinsic factors (for example, behavioural response) and context-specific or moderator factors (for example, site-specific and time-specific impacts, research methods).

Research synthesis does not only have a science-intrinsic benefit. It may also lead to major efficiency gains in empirical research through the possibility of (partial) transferability of practical results, for example for economic assessment or macroeconomic forecasting. Benefit or value transfer ${ }^{1}$ may essentially be conceived as conditional forecasting where the parameters used for inferring statements on future states of a phenomenon under investigation can be derived from information synthesis (such as metaanalytical experiments). Recent applications in this field can be found especially in environmental economics, viz., benefit transfer or value transfer (Button and Nijkamp, 1999; Johnson and Button, 1997; Loomis, 1992).

In the light of the above background remarks, the aim of the present chapter is to offer a further reflection on comparative assessment of scientific knowledge by addressing the potentials of meta-analysis (in section 2). In section 3, we will give a concise introduction to some operational meta-analytical methods and empirical applications. Section 4 discusses the use of scientific knowledge from case studies for value or benefit transfer. The chapter concludes in section 5 with the outlook for further research.

\section{THE POTENTIAL OF META-ANALYSIS}

How much scientific effort is required to reach a certain goal? It is surprising that in basic research this question is hardly raised or answered. But essentially this is an economic question, where the allocation of scarce human resources is at stake. Against this background, it does not seem unrealistic to seek an optimal level of scientific effort in a research process. In principle, one would have to look for an optimal control model with learning effects. In other words, we may investigate whether it is possible to 
infer certain scientific conclusions while economizing on the input side of scarce intellect or creativity during a research process. It is by no means certain that more studies will ultimately lead to more or better insights, as there may be decreasing (or even negative) marginal benefits (due to diseconomies and redundancy). In a statistical sense, this question is often referred to as optimal data sampling, but especially in a more general sense it is intriguing. Do additional studies add to a better understanding of largely similar phenomena? Or in economic terms: do the additional scientific benefits of an extra case study outweigh the marginal costs?

Columbus provides a good example. When he asked Queen Isabella for financial support for his exploration of the New World, he needed to justify his wanting three ships. He could convincingly argue that one ship would be too risky, whereas twenty ships would create serious logistic management problems. Thus, out of a set of rivalry hypotheses on the size of this research endeavour, he had to identify the optimal fleet size (van Doren, 1991).

Another example is provided by Jenner in his experiments into inoculation against smallpox. In 1798, he described how he inoculated twenty patients with smallpox virus to ascertain whether their previous exposure to cowpox gave them protection against smallpox. If we assume (a) that these twenty patients were a random sample who had had cowpox but not smallpox, and (b) that people who had never had smallpox would develop the disease on average nine times out of ten when inoculated with smallpox (and conversely that one in ten times there would be no reaction because of inactive inoculation material or inadequate inoculation technique), then the likelihood of not one of them developing smallpox is $0 \cdot 1^{20}$ or one chance in 100000000000000000000 . Thus Jenner was quite safe in ignoring the possibility that his results could have been due to chance (Langley, 1970) and extra case studies or trials were clearly unnecessary, and would not increase understanding of this phenomenon.

A final illustration can be found in Jane Jacobs' fascinating study, The Death and Life of Great American Cities (1961). Her book addresses survival conditions of American cities, but in her empirical case it sufficed to address mainly New York City as the archetype of other American cities. Thus, although her analysis centred on New York City, she offered the building blocks for a comprehensive general theory on urban dynamics. Her analysis of the critical success and survival conditions of modern cities - sometimes in an anecdotal way - most likely would not have gained so much momentum and conviction, if she had studied all American cities.

Clearly, case study research is essentially research that focuses on the inference of general or transferable findings and is thus also a matter of optimal experimentation (for details, see Yin, 1994). A major problem 
inherent in social science research, however, is a lack of controlled experimentation in empirical investigations. A common design of case studies in different countries is usually lacking, implying that varying ceteris paribus conditions are assumed in distinct case studies. At best, case studies may address the same phenomenon, use more or less the same research methodology or employ to a large extent similar data. This, of course, makes a rigorous synthesis difficult, while also the degree of transferability may sometimes be questionable. This particularly is true when case studies that were never meant to be integrated in a meta-analytical experiment are pooled.

The conditions for a proper application of conventional statistical metaanalysis are fairly stringent. After its genesis in medicine and the natural sciences, meta-analysis was introduced to social science research in the 1970s to overcome common application problems, such as the lack of large data sets to derive general results and the problem of uncertainty of information and of data values. Meta-analysis is a systematic framework that synthesizes and compares past studies, and extends and re-examines the results of the available data to produce more general results than earlier attempts have been able to do, by focusing on a joint kernel of previously undertaken research.

The meta-analysis approach thus offers a series of techniques on measurable phenomena that permits a quantitative aggregation of results across different studies. In so doing, it may help, for example, to generate more clearly numerical values of the economic costs and benefits from the available data. It can also act as a supplement to more common literarytype approaches when reviewing the usefulness of parameters derived from prior studies and help direct new research to related areas. And finally, it may also help to understand the robustness of certain findings by referring to research synthesis as a kind of sensitivity analysis.

The introduction of meta-analysis as a formal procedure for analysing problems has emerged from the necessity to summarize and induce general results from studies already developed on similar problems. Meta-analysis is therefore concerned with the synthesis of results and findings from scientific studies. Glass, who in 1976 coined the term 'meta-analysis', provides a simple definition of this approach: 'Meta-analysis refers to the statistical analysis of a large collection of results from individual studies for the purpose of integrating the findings. It connotes a rigorous alternative to the casual, narrative discussions of research studies which typify our attempt to make sense of the rapidly expanding research literature.'

A commonly used method in social science research is meta-regression analysis. Such a statistical technique has been widely applied in biometrics and sociometrics with rather successful results. Since this approach uses a 
statistical tool, the input data must be quantitative data. The primary characteristics of a meta-regression analysis are the same as those used in standard regression analysis: the statistical link between one of the variables (the dependent variable) by means of other variables (the independent variables). The main problem here is, of course, the variance in the original underlying case study data.

Let us, for the sake of illustration, consider a number of studies which have addressed a common research problem, albeit in different contexts and with different data. For instance, we may address the problem of congestion in commuting traffic. We may then consider studies on transport congestion, which have been conducted in various countries at different times and with different samples. In general, the application of the metaanalysis methodology is used when there is a small set of case studies from which it is difficult to extract a general conclusion. Therefore we combine different studies on the same topic in order to reach a more general conclusion. We can, so to speak, conceive meta-analysis as a puzzle where each piece does not represent the entire figure, but together the pieces make up an integral picture. This picture may be more cohesive if the phenomena under investigation are not particularly study-specific. In addition, in a policy problem, for example, meta-analysis can be applied to reach a balanced decision in the present, based upon prior decisions. To do so, it attempts to define the relationship between cause and effect in the problem under investigation. The general statistical form of a meta-analytical problem will then be as follows:

$$
Y=f(P, X, R, T, L)+\varepsilon,
$$

where $Y$ is the variable under study which has been the focal point of the prior studies under scrutiny, $P$ is what we consider to be the set of causes of the outcome $Y, X$ represents the characteristics of the set of objects under examination affected by $P$ in order to determine the outcome $Y, R$ represents the characteristics of the research methods used in each study (for example, econometric analysis or survey analysis) and the data (for example, time series or cross section), $T$ indicates the time period covered by each study in order to examine time dependency, $L$ expresses the location where each study has been carried out and $\varepsilon$ is the usual error term.

In relation to the types of studies considered here, all of these variables are supposed to have a relative importance in our analysis. For instance, in the field of medical studies where the majority of the studies are experiments in a closed system with the same methodology, attention is mainly focused on the variables $P$ and $X$.

The application of a meta-regression analysis - addressing average 
values of parameter values originating from different studies - can then generate the meaningful comparative results we want to achieve in our survey analysis. Having obtained the regression results, we must carry out various tests that may verify the correctness of our results. Such tests generally aim to assess the effect sizes in the study examined and the accuracy of the results. For instance, we can test how the indicator chosen to reveal the effects of the problem under scrutiny depends upon the design of the research, or how different estimates can be combined into one estimate of the effect size. The most frequently used statistical procedures are the following (see also Bal and Nijkamp, 2001):

1. estimation of individual effect sizes - this is an examination of the correlation of the 'policy' applied and the observed effect, based on standard regression methods;

2. vote counting - this is a procedure which assesses whether a specific effect exists or not, depending on the relative size of the case studies;

3. combined significance - this test aims to reach a conclusion concerning the occurrence of the effect under scrutiny by investigating the validity of the underlying case studies;

4. combining effect sizes and a test on homogeneity - in this test, attention is given to the question of how different estimates can be combined into one estimate of the effect size; and

5. analysing effect sizes - in this test, the variations among the estimated effect sizes are estimated.

Having followed these statistical procedures, we will have, not only a response to the assessment problem concerned, but also a more comprehensive understanding of the characteristics and limitations of the methodology adopted.

In the case of a (statistically based) meta-regression analysis, the data that need to be collected must be quantitative data. Given this condition, a general guideline for deciding whether or not a particular study should be considered in a meta-analytical formulation is based on commonality in research issues. Therefore a meta-regression analysis rests upon the following general rules: the study to be included must focus on the same phenomenon, it must use the same outcome measure and the same population characteristics and, finally, it must have a similar research objective. The problem of the selection of the studies is closely linked to the selection criteria that are needed to identify relevant studies. With regard to these criteria for the selection, particular care must be taken to ensure similarity among the studies. Moreover, we need to verify uniformity and standardization in order to minimize possible errors in the calculation. To avoid this 
problem, it may be necessary to conduct further experiments or to carry out new elaborations and estimations of the data presented in the individual studies (van den Bergh et al., 1997).

Thanks to its specific synthesis potential, a statistical meta-analysis approach may be a useful and rigorous substitute for the standard type of literature review of distinct case studies. Therefore it can assume a relevant role in an initial phase of a study, because such a technique has the capacity to pinpoint aspects of a problem not immediately evident from a cursory examination of data. In the next section we will offer a broader scope for meta-analysis by emphasizing its potential in comparative studies.

\section{META-ANALYTICAL METHODS AND APPLICATIONS}

There may be various methods for research synthesis and comparison. It is increasingly recognized that meta-analysis is not a single statistical method, but a mode of thinking (on research) in order to bring together research findings from different studies with a view to comparison, synthesis, knowledge acquisition, transferability or generalization. Meta-regression is only one such method, but in practice a variety of approaches is possible. We will offer a concise overview here.

In the case of nominal information (that is, the lowest measurement level), the variable under consideration from different studies does not have a numerical meaning, but we can count the frequency of occurrence of certain attributes and link them to other attributes. In this context, contingency table analysis and log-linear modelling may offer meaningful analytical assistance. Verbal and substantive information synthesis used, for instance, in content analysis is often deployed in communication and media studies (Hogenraad, 1989).

If we have categorical information (for example, from surveys), standard approaches such as multinomial logit analysis may be used to infer statistically valid results from a comparative analysis of case studies. Clearly, the sample should be sufficiently large to warrant this approach. Otherwise, soft-modelling approaches would have to be applied (Nijkamp et al., 1984).

Next, the outcomes of various previous case studies may also be measured as ordinal rankings. In such cases, rank correlation analysis may be helpful and, if the sample is sufficiently large, also ordinal meta-regression analysis. An alternative way of comparing ordinal outcomes of different case studies is the use of qualitative multi-criteria analysis, for example, concordance analysis or regime analysis (for a survey, see Nijkamp et al., 1992). 
If the study findings of previous investigations are measured in a cardinal metric (either on an interval scale or a ratio scale), the above-mentioned meta-regression analysis may be a meaningful method. If the sample of case studies is not large enough, one may also employ normal correlation analysis or discriminating analysis.

Finally, there may also be cases where the outcomes of case studies are only linguistically measured, for example, as a fuzzy expression. In that case, use can be made of fuzzy set analysis, in particular if there is a distinct classification with a numerical meaning of the variables (or classes). For a further discussion on fuzzy set analysis for comparative purposes, we refer to Munda (1996). Finally, there is the possibility that the performance of case studies is measured as class information, with distinct class sizes. In such cases rough set analysis appears to be a very powerful tool. For application of rough set analysis, we refer to van den Bergh et al. (1997), and Nijkamp and Ursem, Chapter 15 of the present volume.

Recently, attempts have been made also to identify common characteristics and differences between case study results and to transfer results for predictive purposes - especially in the framework of land-use planning, tourism and environmental management - by introducing Geographic Information Systems (GIS). We refer to Bateman et al. (1998) for further details.

In conclusion, there is a wide variety of meta-analytical approaches. All of them aim to shed light on the key findings of various studies in order to synthesize the most prominent results from earlier research work. In recent years, we have seen a steady growth in meta-analytical studies in economics, in particular in the area of regional, transport and environmental economics. Here we will illustrate the research potential of meta-analysis by referring to various studies undertaken in the above fields.

In the area of regional economics, meta-analytical studies have been undertaken, inter alia, in the assessment of regional tourist multipliers in order to derive the best possible estimate of a regional tourist income multiplier for a given tourist area on the basis of empirically estimated multiplier values for various other tourist destinations (Baaijens and Nijkamp, 2000) and the identification of critical success factors for spatial sustainability policy with the aim of identifying the most important impediments and opportunities of urban environmental sustainability initiatives (Nijkamp and Pepping, 1998a). Techniques used in these applications were, inter alia, meta-regression analysis, discrete choice modelling and rough set theory.

In the transport field, various meta-analytical applications also can be found. Examples are the estimation of the variance in public transport demand elasticity in various European countries based on a synthesis of previously undertaken case studies (Nijkamp and Pepping, 1998b) and studies on congestion policy based on an assessment of the effectiveness of 
traffic restraint polices (Button and Kerr, 1996). The meta-analytical methods used in these studies again comprise meta-regression analysis, correlation analysis and rough set theory.

Finally, in environmental economic research, we have witnessed a great variety of meta-analytical studies. Examples are the evaluation of contingent valuation methods for air pollution (Smith and Huang, 1993, 1995) and noise nuisance (Button, 1995). Also the methods deployed were meta-regression analysis and correlation analysis. It appears that meta-analysis has become an established approach in environmental analysis and management, with various applications of contingent valuation, travel cost and hedonic pricing methods. The same applies to the related research area of recreation analysis (Brouwer et al., 1997; Smith and Karou, 1990; Walsh et al., 1990, 1992).

The field of meta-analytical research is indeed a rapidly growing field, and one of the interesting applications is the combination of meta-analysis with value or benefit transfer.

\section{VALUE OR BENEFIT TRANSFER}

Value or benefit transfer is a technique in which the results of studies performed earlier are applied to new policy contexts (Brouwer, 2000). Three main types of value transfer can be distinguished: (a) simple transfer of a mean effect size (for instance, a willingness to pay estimate, or an elasticity), (b) transfer of a demand or bid function (that is, benefit function transfer), and (c) transfer of an estimate based on a meta-analysis.

Within the realm of environmental and natural resource economics value transfer is now increasingly recognized as a viable technique. Much of the early work can be found in a special issue of Water Resources Research (1992, vol. 28, number 3; see also the discussion in Ecological Economics, 1998, vol. 25, number 1) and is mainly concerned with the transfer of values of natural resources. The latter are typically not traded in a market setting, and hence contingent valuation, hedonic pricing and travel cost methods are used to assess the benefits associated with policy changes. ${ }^{2}$

Boyle and Bergstrom (1992) present a very readable overview of the practice and associated problems with value transfer. Luken et al. (1992) and Desvouges et al. (1992) used benefit function transfer to estimate the impact of effluents discharge from pulp and paper mills into rivers. Kask and Shogren (1994) assessed long-term health risks associated with surface water contamination. In addition, they developed a benefit transfer protocol. The work of Costanza et al. (1997) was received with considerable scepticism and further stimulated the discussion about the validity and accuracy of value transfer. 
Early studies by Loomis (1992) and Smith (1992) had already focused on the margin of error in transferring estimated values from one site (the socalled 'study site') to the policy site. Loomis (1992) attained mixed results, and Smith (1992) demonstrated that estimated benefits associated with limiting effluents even turn into a net-cost situation under value transfer. Downing and Ozuna (1996) added further doubts to the accuracy of value transfer, in looking at the recreational value of salt-water angling, showing that transfer errors are substantial. Some authors attribute the occurrence of substantial transfer errors to the lack of a standardized protocol, others are much more critical of the entire set-up.

In sum, the value transfer technique is very appealing, mainly because it has the potential to economize on the available knowledge stock. This is generally appreciated by policy makers, because they are usually interested in attaining quantitative policy advice at low cost. The technique is, however, also very controversial. A main reason for the scepticism is that the transfer errors are rather substantial. Further evidence in that respect can be found in the contributions to this volume by Bergland et al. (Chapter 7) and Engel (Chapter 8). Engel explicitly considers the role of meta-analysis with respect to the accuracy and reliability of value transfer.

\section{PROSPECTS OF META-ANALYSIS AND VALUE TRANSFER}

Meta-analysis has turned into a research style that aims to combine generality and specificity (Espey 1996). It may be a cost-effective and expeditious way of economizing on research efforts through the focus on main determinants of a phenomenon, seen from a comparative perspective. The same applies to value and benefit transfer studies. Clearly, there is need for some caution: the moderator variables have to be carefully investigated and proper care regarding study and context-specificity is warranted. Against this background, the use of the ceteris paribus clause also deserves due attention, as this is essentially a non-controlled black box (Bal and Nijkamp, 2001; Persky, 1990).

Further evidence is needed with respect to the validity and reliability of value transfer. Until now, the analysis of the performance of value transfer has mainly focused on the comparison of mean values. As more and more results become available, the comparison may be cast in a statistical setting. Furthermore, value transfer has until now largely been limited to the field of the valuation of natural resources, which is difficult in itself because of the non-market setting. The transfer of values developed in a market setting, such as tourist multipliers (Baaijens and Nijkamp, 2000) or water 
demand elasticities (Dalhuisen et al., 2001), may be more straightforward as well as more accurate.

A new field where meta-analysis may likely play an interesting role is micro-based survey research. To some extent, the collection and description of the attributes of a given individual may be conceived as a detailed case study focused on one person. By combining the survey results on multiple persons - while addressing the same research issues across the entire sample of individuals - the response variables can be treated and explained in the same way as in a standard meta-analysis (including, for instance, the correction for context-specific factors).

Another area where meta-analysis can usefully be deployed is a review of reviews; in other words, a meta-study on meta-analyses. Of course, this requires a sufficiently large sample of meta-studies, but in some disciplines such meta-studies do exist: for example, in educational sciences. A good example can be found in Becker (1998), who classified meta-studies systematically into instructional books, methodological books and applicationoriented books. Each of these classes of meta-studies was then investigated in more detail, albeit only in a literary form.

Finally, there may be new perspectives for meta-analysis if it is linked to Bayesian statistics. If previous case studies are regarded as sources of prior information, adjusted Bayesian tools may be applied (Lubbe, 1998; see also Brunsdon and Willis, in Chapter 11 of the present volume).

In combination with large databases and improved access to computerized information systems, meta-analysis will most likely become an important tool in normal research practice. In addition to a synthesis of knowledge, it will increasingly be used in a deductive science methodology as well as in the applied field of value transfer with a view to the identification of bandwidths in the variation of phenomena.

\section{NOTES}

1. We use the terms interchangeably, although one can argue that 'value transfer' is the more neutral term, as compared to 'benefit transfer' (see Heijungs' remark in Chapter 2 of the present volume and Brouwer, 2000).

2. This is the main reason why the technique is frequently referred to as 'benefit' transfer instead of the more neutral 'value' transfer.

\section{REFERENCES}

Baaijens, S. and P. Nijkamp (2000), Meta-analytic Methods for Comparative and Exploratory Policy Research', Journal of Policy Modelling, 22, 821-58. 
Bateman, I.J., J.S. Brainard and A.A. Lovett (1998), 'Modelling Woodland Recreation Demand Using Geographical Information Systems: A Benefit Transfer Study', CSERGE Working Paper GEC 95-06, University of East Anglia, Norwich.

Becker, B.J. (1998), Mega-review: Books on Meta-analysis', Journal of Educational and Behavioural Statistics, 23, 77-92.

Bergh, J.C.J.M. van den, K. Button, P. Nijkamp and G. Pepping (1997), Metaanalysis in Environmental Economics, Dordrecht: Kluwer.

Boyle, K.J. and J.C. Bergstrom (1992), 'Benefit Transfer Studies: Myths, Pragmatism and Idealism', Water Resources Research, 28, 657-63.

Brouwer, R. (2000), 'Environmental Value Transfer: State of the Art and Future Prospects', Ecological Economics, 32, 137-52.

Brouwer, R., I.H. Langford, I.J. Bateman, T.C. Crowards and R.K. Turner (1997), 'A Meta-analysis of Wetland Contingent Valuation Studies', CSERGE Working Paper GEC 97-20, Centre for Social and Economic Research on the Global Environment (CSERGE), University of East Anglia and University College London.

Button, K.J. (1995), 'What can Meta-analysis Tell us about the Implications of Transport?', Regional Studies, 29, 507-17.

Button, K.J. and J. Kerr (1996), 'Effectiveness of Traffic Restraint Policies: A Simple Meta-regression Analysis', International Journal of Transport Economics, 23, 214-35.

Button, K. and P. Nijkamp (1999), 'The Realities of Using "Benefit Transfers" in Transport Decision-Making', research paper, Tinbergen Institute, Amsterdam.

Button, K., F. Bal and P. Nijkamp (1999), 'Ceteris Paribus, Meta-analysis and Value Transfer', research paper, Tinbergen Institute, Amsterdam.

Costanza, R., R. d'Arge, R. de Groot, S. Farber, M. Grasso, B. Hannon, K. Limburg, S. Naeem, R.V. O'Neill, J. Paruelo, R.G. Raskin, P. Sutton and M. van den Belt (1997), 'The Value of the World's Ecosystem Services and Natural Capital', Nature, 387, 253-60.

Dalhuisen, J.M., R.J.G.M. Florax, H.L.F. de Groot and P. Nijkamp (2001), 'Price and Income Elasticities of Residential Water Demand: Why Empirical Estimates Differ', research paper, Tinbergen Institute, Amsterdam.

Desvousges, W.H., M.C. Naughton and G.R. Parsons (1992), 'Benefit Transfer: Conceptual Problems in Estimating Water Quality Benefits Using Existing Studies', Water Resources Research, 28, 675-83.

Doren, Ch. van (1991), A History of Knowledge, New York: Ballantine.

Downing, M. and T. Ozuna Jr (1996), 'Testing the Reliability of the Benefit Function Transfer Approach', Journal of Environmental Economics and Management, 30, 316-22.

Espey, M. (1996), 'Explaining the Variation in Elasticity Estimates of Gasoline Demand in the United States: A Meta-analysis', The Energy Journal, 17, 49-60.

Glass, G.V. (1976), 'Primary, Secondary and Meta-analysis of Research', Educational Researcher, 5, 3-8.

Hogenraad, R. (1989), 'The Rhetoric Texture of an Ad', in G. Morello (ed.), Consumer Behaviour and Strategic Marketing, Palermo: ISIDA.

Jacobs, J. (1977), The Death and Life of Great American Cities, Harmondsworth: Penguin; first published 1961.

Johnson, K. and K. Button (1997), 'Benefit Transfers: Are They a Satisfactory Input to Benefit Cost Analysis?', Transportation Research D, 2, 223-31. 
Kask, S.B. and J.F. Shogren (1994), 'Benefit Transfer Protocol for Long-Term Health Risk Valuation: A Case of Surface Water Contamination', Water Resources Research, 30, 2813-23.

Langley, R. (1970), Practical Statistics for Non-Mathematical People, London: Pan Books.

Loomis, J.B. (1992), 'The Evolution of a More Rigorous Approach to Benefit Transfer: Benefit Function Transfer', Water Resources Research, 28, 701-5.

Lubbe, M. (1998), 'Van Remmend Naar Prikkelend, Marktwerking in de Sociale Zekerheid', MA thesis, Department of Spatial Economics, Free University, Amsterdam.

Luken, R.A., F.R. Johnson and V. Kibler (1992), 'Benefits and Costs of Pulp and Paper Effluent Controls under the Clean Water Act', Water Resources Research, 28, 665-74.

Munda, G. (1996), Fuzzy Information in Multicriteria Environmental Evaluation Models, Heidelberg: Physica-Verlag.

Nijkamp, P. and G. Pepping (1998a), 'A Meta-analysis of Sustainable City Initiatives', Urban Studies, 35, 1481-1500.

Nijkamp, P. and G. Pepping (1998b), 'A Meta-analysis for Explaining the Variance in Public Transport Demand Elasticities in Europe', Journal of Transportation and Statistics, 1, 1-14.

Nijkamp, P. and A. Reggiani (1998), The Economics of Complex Spatial Systems, Amsterdam: Elsevier.

Nijkamp, P., H. Leitner and N. Wrigley (eds) (1984), Measuring the Unmeasurable, Dordrecht: Kluwer.

Nijkamp, P., P. Rietveld and H. Voogd (1992), Multi-criteria Analysis in Physical Planning, Amsterdam: North-Holland Elsevier.

Persky, J. (1990), 'Retrospectives; Ceteris Paribus', Journal of Economic Perspectives, 4, 187-93.

Smith, V.K. (1992), 'On Separating Defensible Benefit Transfers from "Smoke and Mirrors", Water Resources Research, 28, 685-94.

Smith, V.K. and J.C. Huang (1993), 'Hedonic Models and Air Pollution: Twentyfive Years and Counting', Environmental and Resource Economics, 3, 381-94.

Smith, V.K. and J.C. Huang (1995), 'Can Markets Value Air Quality?', Journal of Political Economy, 103, 209-27.

Smith, V.K. and Y. Karou (1990), 'Signals or Noise? Explaining the Variation in Recreation Benefit Estimates', American Journal of Agricultural Economics, 72, 419-33.

Walsh, R.G., D.M. Johnson and J.R. McKean (1990), 'Nonmarket Values from Two Decades of Research on Recreation Demand', Advances in Applied Microeconomics, 5, 167-93.

Walsh, R.G., D.M. Johnson and J.R. McKean (1992), 'Benefit Transfer of Outdoor Recreation Demand Studies, 1968-1988', Water Resources Research, 28, 707-14.

Yin, R.K. (1994), Case Study Research, London: Sage. 